\title{
B-glucuronidase of family-2 glycosyl hydrolase: A missing member in plants
}

\author{
Loganathan Arul*, George Benita, Duraialagaraja Sudhakar, Balsamy Thayumanavan and Ponnusamy \\ Balasubramanian \\ ${ }^{1}$ Centre for Plant Molecular Biology, Tamil Nadu Agricultural University, Coimbatore 641003, India; \\ Loganathan Arul - Email: arulsra@gmail.com; Phone: 91422 6611353; * Corresponding author \\ received November 25, 2008; accepted December 01, 2008; published December 31, 2008
}

\begin{abstract}
:
Glycosyl hydrolases hydrolyze the glycosidic bond in carbohydrates or between a carbohydrate and a non-carbohydrate moiety. $\beta$ - glucuronidase (GUS) is classified under two glycosyl hydrolase families ( 2 and 79 ) and the family- $2 \beta$-glucuronidase is reported in a wide range of organisms, but not in plants. The family-79 endo- $\beta$-glucuronidase (heparanase) is reported in microorganisms, vertebrates and plants. The E. coli family- $2 \beta$-glucuronidase (uidA) had been successfully devised as a reporter gene in plant transformation on the basis that plants do not have homologous GUS activity. On the contrary, histochemical staining with XGluc was reported in wild type (non-transgenic) plants. Data shows that, family- $2 \beta$-glucuronidase homologous sequence is not found in plants. Further, $\beta$-glucuronidases of family-2 and 79 lack appreciable sequence similarity. However, the catalytic site residues, glutamic acid and tyrosine of the family- $2 \beta$-glucuronidase are found to be conserved in family-79 $\beta$-glucuronidase of plants. This led to propose that the GUS staining reported in wild type plants is largely because of the broad substrate specificity of family-79 $\beta$-glucuronidase on X-Gluc and not due to the family-2 $\beta$-glucuronidase, as the latter has been found to be missing in plants.
\end{abstract}

Keywords: glycosyl hydrolase; $\beta$-glucuronidase; heparanase; endogenous GUS activity

\section{Background:}

Glycosyl hydrolases (GH) are a widespread group of enzymes hydrolyzing the glycosidic bond between two or more carbohydrates or between a carbohydrate and a noncarbohydrate moiety. GH was classified traditionally based on their substrate specificity. However, the classification based on substrate specificity was complicated by the fact that some enzymes hydrolyze more than one substrate. This resulted in reorganization of glycosyl hydrolases into families based on amino acid sequence similarities and intends to better reflect the structural features of these enzymes than their sole substrate specificity [1]. At present there are about $113 \mathrm{GH}$ families that are available on the continuously updated Carbohydrate Active enZYmes (CAZy) web server [2]. $\beta$-glucuronidase (GUS) is a well characterized glycosyl hydrolase classified under two $\mathrm{GH}$ families, 2 and 79 respectively (CAZy). The enzyme catalyzes the hydrolysis of $\beta$-glucuronic acid residues from the non-reducing termini of glycosaminoglycans (GAGs). The reaction catalyzed by $\beta$ glucuronidase is, $\beta$-D-glucuronide $+\mathrm{H}_{2} \mathrm{O} \rightarrow$ D-glucuronate + alcohol.

The family-2 $\beta$-glucuronidase sequence was reported in a wide range of organisms, this include Homo sapiens, Rattus norvegicus, Drosophila melanogaster, Penicillium canescens and Escherichia coli. However, family-2 $\beta$-glucuronidase sequence has been so far not reported in plants. The family$79 \beta$-glucuronidase had been characterized in Homo sapien and was known as heparanase, it is involved in the

ISSN 0973-2063 (online) 0973-8894 (print)

Bioinformation 3(5): 194-197 (2008) hydrolysis of heparan sulfate, a proteoglycan [3]. Apart from vertebrates, heparanase homologous sequence had been reported in a few microorganisms and in plants as well (Scutellaria baicalensis, Arabidopsis thaliana, Oryza sativa, Hordeum vulgare and Medicago truncatula). Referring to family-2 glucuronidase, the E. coil GUS encoded by (uidA) had been used for the development of a reporter gene system for plant transformation experiments [4]. Transgenic plants expressing E. coli GUS resulted in histochemical staining with the substrate 5-Bromo-4-chloro-3-indolyl-beta-Dglucuronide (X-Gluc) on GUS assay at the physiological $\mathrm{pH}$ (7.0). The enzyme GUS cleaved the $\beta$-glucuronic acid from the substrate X-Gluc and resulted in the development of a blue color of the transformed tissues. However, the untransformed/wild type plant remained unstained due to the absence of uidA activity. Jefferson and coworkers had also reported the absence of endogenous GUS activity in a broad spectrum of higher plants [5]. On the contrary, endogenous GUS activity evidenced by blue staining with X-Gluc had been reported in wild type (untransformed) plants particularly in immature tissues of a few species. The GUS activity was found to be present in male gametophyte of several solanaceous plants [6]. Similarly an extensive study of 52 seed-plants species showed that plants lack GUS activity in vegetative organs but has activity in reproductive tissues [7]. Alwen and coworkers reported endogenous GUS activity at pH 5.0 in a wide variety of plant species [8]. Further, the 194 
vegetative and floral organs of Arabidopsis thaliana at $\mathrm{pH}$ 5.0 [9]. Above all, characterization of endogenous GUS activity in selected models such as Arabidopsis thaliana, Oryza sativa, Zea mays and Nicotiana tobaccum clearly demonstrated the ubiquitous presence of GUS activity at an optimum $\mathrm{pH}$ of 4.0 [10]. In the light of reports on histochemical staining in wild type plants, the present study aim to resolve the basis for GUS staining in plants. We took to bioinformatics approach and analyzed the amino acid sequences of $\beta$-glucuronidases from family- 2 and family- 79 . As an outcome of this study, we propose the absence of family-2 $\beta$-glucuronidase in plants and implicate family-79 $\beta$-glucuronidase (heparanase) for the alleged histochemical activity in wild type plants.

\section{Methodology:}

The GeneBank and GenPept databases of National Centre for Biotechnology Information (NCBI) and the Carbohydrate-
Active enZYmes server (CaZY) were accessed for this study [11]. Amino acid sequence of $\beta$-glucuronidase (family-2): Homo sapiens (NP_000172), Escherichia coli K12 (AAC74689), Penicillium canescens (AAV91787) and $\beta$ glucuronidase (family-79): Homo sapiens (AAD54516), Scutellaria baicalensis (BAA97804), Arabidopsis thaliana (BAB09947), Oryza sativa (BAD36026), Hordeum vulgare (CAD42650), Medicago truncatula (ABE93308) were downloaded from GenPept. Pairwise sequence alignment was done using align program with BLOSUM62 matrix [12]. The search for $\beta$-glucuronidase (family-2) homolog in plants was performed with PSI-BLAST and TBLASTN programs [13]. Multiple sequence alignment of the heparanase sequences was done using ClustalW [14]. The chemical structures of XGluc and Baicalein 7-O- $\beta$-D-glucuronide were retrieved from PubChem database [15].

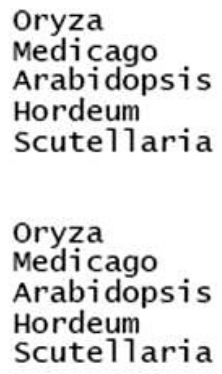

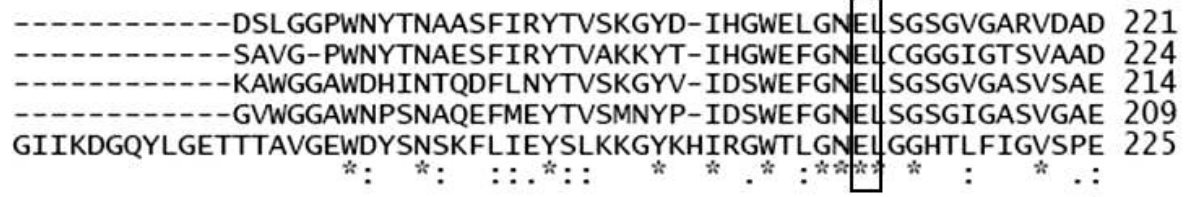

QYAQDVLALKQIIDNSYQG-HASKPLVIAPGGFFDAAWFTELISRTKPNQMDVMTHHIYN 280 QYASDVIVLRKIIQDVYRG-VKPKPLIIAPGGFFDENWFKEFLNKSD-ESTNVVTHHIYN 282 LYGKDLIVLKDVINKVYKNSWLHKPILVAPGGFYEQQWYTKLLEISGPSVVDVVTHHIYN 274 QYGKDLVELQKIVDQLYEN--PSKPLVLAPGGFYDKQCFAQLLDVSGPNVVRGMTHHIYN 267 DYANDAKKLHELVKEIYQD-QGTMPLIIAPGAIFDLEWYTEFIDRTP--ELHVATHHMYN 282

Figure 1: A segment of the multiple sequence alignment of plant heparanase from Oryza sativa, Medicago truncatula, Arabidopsis thaliana, Hordeum vulgare and Scutellaria baicalensis is shown. The conserved catalytic site residues (glutamic acid and tyrosine) are shown in boxes.

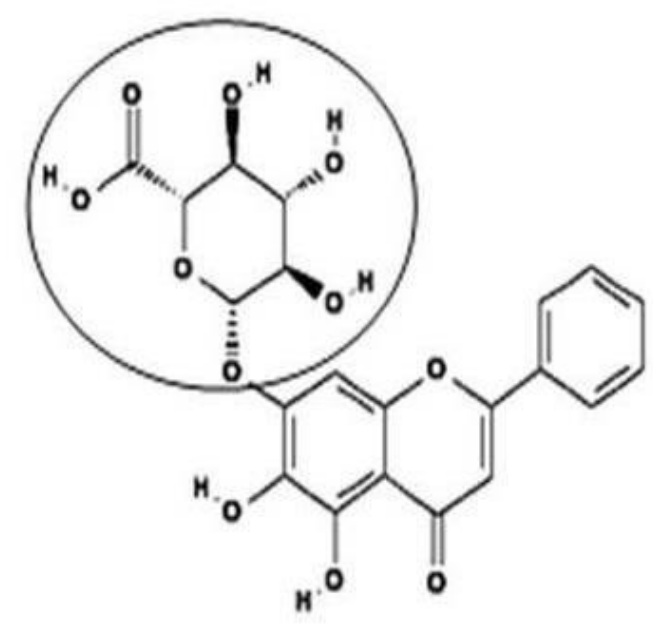

a

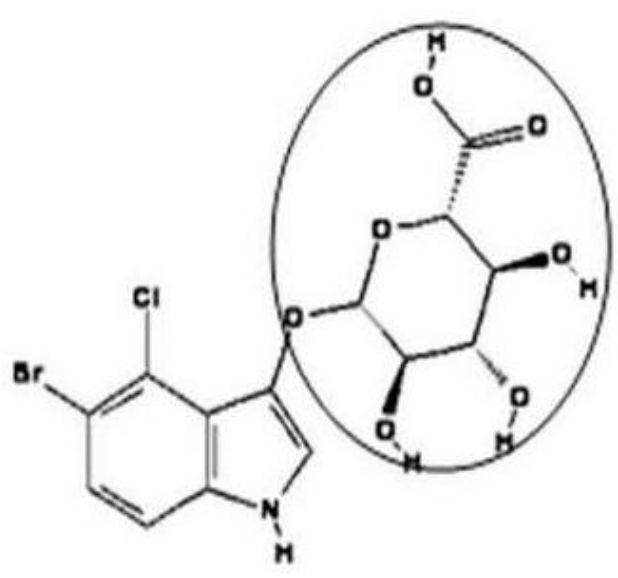

b

Figure 2: Structure of (a) Baicalein 7-O- $\beta$-D-glucuronide and (b) X-Gluc showing the glucuronate moiety present in common (encircled).

ISSN 0973-2063 (online) 0973-8894 (print) 


\author{
www.bioinformation.net
}

\section{Hypothesis}

\begin{abstract}
Discussion:
$\beta$-glucuronidase sequences belonging to family-2 and 79 reflected the existence of only a weak similarity/identity at the amino acid level. Pairwise alignment of human heparanase (family-79) and human $\beta$-glucuronidase (family2) showed only $11.4 \%$ identity and $18.7 \%$ similarity. Similarly, human heparanase and E. coli $\beta$-glucuronidase (family-2) had only $11.7 \%$ identity and $22.0 \%$ similarity. In contrast, human and $E$. coli $\beta$-glucuronidase of family- 2 shared significant level of identity $(42.6 \%)$ and similarity $(55.6 \%)$ despite their distance of separation in evolution. This impelled us to note that $\beta$-glucuronidase classified under the glycosyl hydrolase families (2 and 79) are two distinct categories of enzymes. Here we wish to discuss the role of $E$. coli family-2 $\beta$-glucuronidase (uidA) as a reporter gene in plant transformation experiments [5]. The alleged absence of homologous GUS activity in plants formed the basis for successfully devising one such system. In fact, till date there have been no reports on gene sequence cloned/annotated to encode for family-2 $\beta$-glucuronidase in plants. However, to belie this, there are a few reports on GUS activity in wild type plants, as revealed by histochemical staining with $\mathrm{X}$ Gluc $[6,7,8,9,10]$. To resolve the basis for GUS staining, we searched the GenBank for plausible nucleotide sequences that are homologous to the members of family-2 $\beta$ glucuronidase in plants. PSI-BLAST which helps to find distantly related members was carried out with family-2 $\beta$ glucuronidase from E. coli, Penicillium, Homo sapiens and other organisms as query, but the search failed to detect corresponding homolog in plants. Subsequently, a translated BLAST (TBLASTN) search was done against the whole genome sequences of Arabidopsis thaliana and Oryza sativa using the same query sequences. However, the search failed to detect homologous sequence of family- $2 \beta$-glucuronidase in the above genomes for which the complete genomic sequence is available in the GenBank.
\end{abstract}

Now that we refer to sGUS of Scutellaria baicalensis, the only well characterized plant endo- $\beta$-glucuronidase belonging to family-79. Sasaki and coworkers reported the cloning of sGUS, a family-79 $\beta$-glucuronidase from the plant Scutellaria baicalensis [16]. sGUS encodes for baicalinase [17] and is required for the initiation of $\mathrm{H}_{2} \mathrm{O}_{2}$ metabolism. It acts on baicalein-7-O- $\beta$-D-glucuronide (the physiological substrate), leading to the formation of baicalein and glucuronate (when Scutellaria cells produced a large amount of $\mathrm{H}_{2} \mathrm{O}_{2}$ in response to different stresses, baicalein 7-O- $\beta$-Dglucuronide gets hydrolyzed into baicalein by baicalinase). As that of human heparanase, sGUS too showed a very weak similarity with the $\beta$-glucuronidase of family-2 (16.4\% and $4.6 \%$ for human and E. coli respectively). However, sGUS shared a high level similarity of $56.3 \%, 56.9 \%, 54.7 \%$ and $61.1 \%$ with other plant heparanase sequence, Arabidopsis thaliana, Oryza sativa, Hordeum vulgare and Medicago truncatula, respectively. Albeit the lack of appreciable sequence similarity between the $\beta$-glucuronidases of family- 2 and family-79, a glutamic acid and tyrosine residue implicated in the catalytic activity of family-2 $\beta$ glucuronidase $[18,19]$ was found to be strongly conserved in sGUS at $\mathrm{Glu}^{212}$ and $\mathrm{Tyr}^{281}[\mathbf{1 6}]$. This hinted that, catalytic site residues likely glutamic acid and tyrosine are conserved across family-2 and family-79 $\beta$-glucuronidases. Hence, we did a multiple sequence alignment for available plant heparanase sequences from Scutellaria baicalensis, Arabidopsis thaliana, Oryza sativa, Hordeum vulgare and Medicago truncatula. A similar conservation of glutamic acid and tyrosine residues was observed in all plant heparanase sequences at positions corresponding to sGUS (Figure 1). This find led to propose that, conserved glutamic acid and tyrosine residues in heparanase are involved in the cleavage of glucuronate moiety from the physiological substrate (e.g) Baicalein 7-O- $\beta$-D-glucuronide. We further extend to state that heparanase does also hydrolyze unintended substrates, such as X-Gluc. The hydrolysis of X-Gluc is hypothesized to have taken place due to the broad substrate specificity of family-79 $\beta$-glucuronidase on X-Gluc under acidic $\mathrm{pH}$, about 4.0. From the chemical structure of Baicalein 7-O- $\beta-D-$ glucuronide and $\mathrm{X}$-Gluc, it is evident that ' $\beta$-glucuronate' is the only common moiety found in the above two structures (Figure 2). Heparanase, may therefore bind either with Baicalein 7-O- $\beta$-D-glucuronide or X-Gluc via the glucuronate moiety and result into hydrolysis of the glycosidic bond. The above hypothesis further qualifies from the fact that glycosyl hydrolases in general were known to possess broader substrate specificity [1].

\section{Conclusion:}

The gene encoding for family-2 $\beta$-glucuronidase has been so far not reported in plants. However, it was known to be present in different groups of organism such as archaebacteria, eubacteria, fungi, invertebrates and vertebrates. Our search to find family-2 $\beta$-glucuronidase homologous sequence in plants turned out to be unsuccessful, suggesting that family-2 GUS could be missing in plants. Further, analyses of family- 2 and family-79 $\beta$-glucuronidases provided a sequence based functional insight on the origin of histochemical staining in wild type plants. The proposed broad substrate action of family-79 $\beta$-glucuronidase on XGluc explains the basis for GUS staining reported in wild type plants at low $\mathrm{pH}$. However, the absence of histochemical staining at physiological $\mathrm{pH}$ is on expected lines, as plant heparanase was known to have acidic $\mathrm{pH}$ for its optimal activity. On the other hand, family- $2 \beta$-glucuronidase works only at $\mathrm{pH} 7.0$ and transgenic plants overexpressing GUS contributes for the histochemical activity, the absence of GUS staining in wild type plants at the physiological $\mathrm{pH}$ confirms that family- $2 \beta$-glucuronidase is missing in plants.

\section{Acknowledgment:}

This work was carried out at the Bioinformatics centre developed with the financial support of the Department of Biotechnology, Government of India under the Biotechnology Information System (BTIS) program. 


\section{www.bioinformation.net}

\section{Hypothesis}

References:

[01] B. Henrissat, Biochem. J., 280: 309 (1991)

[02] B. Henrissat and A. Bairoch, Biochem. J., 316: 695 (1996)

[03] M. Nakajima et al., J. Biol. Chem., 259: 2283 (1984)

[04] R. A. Jefferson et al., Proc. Natl. Acad. Sci., 83: 8447 (1986)

[05] R. A. Jefferson et al., Euro. Mol. Biol. J., 6: 3901 (1987)

[06] L. Plegt and R. J. Bino, Mol. Gen. Genet., 216: 321 (1989)

[07] C. Y. Hu et al., Plant Cell Rep., 9: 1 (1990)

$\begin{array}{ll}\text { [08] } & \text { A. Alwen et al., Transgen. Res., 1: 63 (1992) } \\ {[\mathbf{0 9}]} & \text { T. Martin et al., Plant Mol. Biol. Rep., 10: } 37 \text { (1992) } \\ {[\mathbf{1 0}]} & \text { C. Sudan et al., Planta., 224: } 853(2006) \\ {[\mathbf{1 1}]} & \text { http://www.cazy.org } \\ {[\mathbf{1 2}]} & \text { http://www.ebi.ac.uk/emboss/align } \\ {[\mathbf{1 3}]} & \text { http://www.ncbi.nlm.nih.gov/BLAST } \\ {[\mathbf{1 4}]} & \text { http://www.ebi.ac.uk/clustalw } \\ {[\mathbf{1 5}]} & \text { http://pubchem.ncbi.nlm.nih.gov } \\ {[\mathbf{1 6}]} & \text { K. Sasaki et al., J. Biol. Chem., 275: } 27466(2000) \\ {[\mathbf{1 7 ]}} & \text { G. A. Levvy et al., Biochem. J., 56: 462 (1954) } \\ {[\mathbf{1 8}]} & \text { S. Jain et al., Nat. Struct. Biol., 3: 375 (1996) } \\ {[\mathbf{1 9}]} & \text { M. R. Islam et al., J. Biol. Chem., 274: 23451 (1999) }\end{array}$

Edited by P. Kangueane

Citation: Arul et al., Bioinformation 3(5): 194-197 (2008)

License statement: This is an open-access article, which permits unrestricted use, distribution, and reproduction in any medium, for non-commercial purposes, provided the original author and source are credited. 